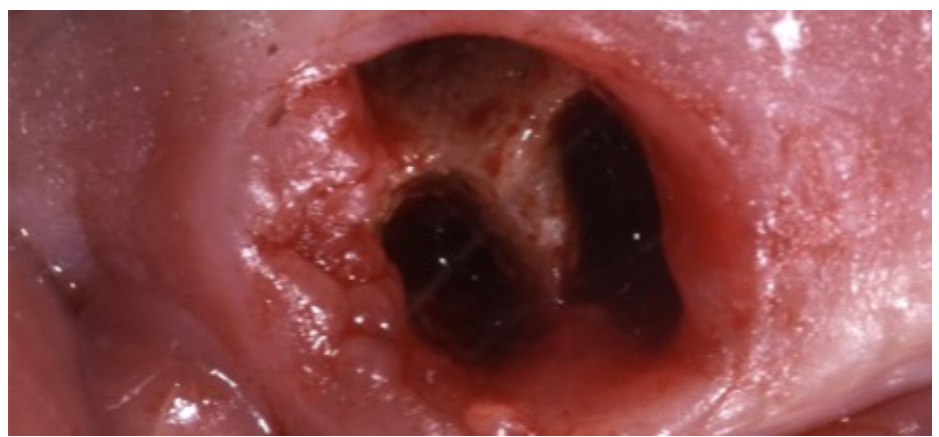

\title{
Histological findings of osteonecrosis spotted prior to tooth extractions. Should we consider tooth extraction still the main trigger event?
}

Rodolfo Mauceri ${ }^{1}$, Vera Panzarella ${ }^{1}$, Martina lurato Carbone ${ }^{1}$, Giacomo Oteri ${ }^{2}$, Antonia Marcianó, olga di fede ${ }^{1}$, Giuseppina Campisi ${ }^{1}$

\section{University of Palermo}

2 University of Messina

Funding: The author(s) received no specific funding for this work.

Potential competing interests: The author(s) declared that no potential competing interests exist.

\section{Abstract}

Osteonecrosis of the jaw $(\mathrm{ONJ})$ is an adverse drug reaction described as the progressive destruction and death of bone that affects the mandible and maxilla of patients exposed to the treatment with medications known to increase the risk of disease, in the absence of a previous radiation treatment.

Tooth extraction often precedes the manifestation of $\mathrm{ONJ}$; indeed, it is sometimes called trigger event and it have also been considered as risk factors for the onset of ONJ.

As a consequence, some of the guidelines recommend avoiding tooth extractions in patients at risk of ONJ; however, a growing body of evidence indicates that dental/periodontal infection prior to extraction, rather than dental extraction may represent the main local risk factor for ONJ.

Ten patients at risk of ONJ have undergone tooth extractions. They were identified and included in our retrospective monocentric clinical investigation.

Patients underwent tooth extractions with standardized procedures (PROMaF protocol), and bone biopsies were taken.

Extractions were performed due to symptomatic, non-restorable teeth in patient at risk of ONJ; histological findings of ONJ were observed in all samples.

This outcome may highlight that the proof of non-exposed ONJ might be the histopathologic confirmation of necrotic 
bone, as stated by European task force on MRONJ. Additionally, alveolar biopsy should possibly be taken in every case of suspected ONJ, which needs an proper and prompt management for successful healing.

\section{Background:}

Osteonecrosis of the jaw $(\mathrm{ONJ})$ is an adverse drug reaction described as the progressive destruction and death of bone that affects the mandible and maxilla of patients exposed to the treatment with medications known to increase the risk of disease, in the absence of a previous radiation treatment ${ }^{[1][2]}$.

Several risk factors have been associated with increased likelihood of ONJ development, including systemic, drug-related and oral risk factors ${ }^{[2][3][4]}$. Among ONJ risk factors, tooth extraction have been described as one of the major risk factors for the onset of $\mathrm{ONJ}$; indeed, it is considered a potential trigger for ONJ ${ }^{[2][3][4]}$.

As a consequence, some of the guidelines recommend avoiding tooth extractions in patients at risk of ONJ; however, a growing body of evidence indicates that dental/periodontal infection prior to extraction, rather than dental extraction may represent the main local risk factor for ONJ ${ }^{[5]}$.

The aim of this retrospective study was to assess the histology of the alveolar bone of cancer patients that underwent dental extractions.

\section{Materials and Methods:}

Study Design

A retrospective monocentric hospital-based study on cancer patients attending the Unit of Oral Medicine of the Paolo Giaccone Policlinico University Hospital in Palermo (Italy) was performed. The study is in accordance with the 2013 Declaration of Helsinki and has been approved by the standing ethical committee of the Paolo Giaccone Policlinico University Hospital in Palermo (Italy).

After clinical and radiological examination, tooth extraction was recommended as the necessary treatment of choice in all cases $^{[2]}$. A standardized medical-surgical protocol for dental extraction application was applied to all patients enrolled. Before surgical procedures, all patients signed written informed consent.

\section{Entry and Exclusion Criteria}

Patients were eligible for the study if they: (I) were aged >18 years; (II) had treatment with ONJ-related drugs because of the underlying disease; (III) required tooth extraction (of non-restorable teeth); (IV) had an absence, at baseline, of unequivocal clinic-radiological signs of ONJ (according to the Italian Societies of Maxillo-Facial Surgery and of Oral Pathology and Medicine) $)^{[2]}$.

Patients were excluded from the study if they: (I) had unequivocal clinical or radiological signs of ONJ in the surgical area; (II) had a previous history of irradiation to the head and neck area; (III) had neoplastic involvement of the jaws; (IV) were in poor general condition; $(\mathrm{V})$ were pregnant or breast-feeding women.

\section{Clinical and Surgical Procedures}


During the first visit, medical, pharmacological, and dental histories of patients were recorded.

All patients underwent peri-operative pharmacological treatment, based on the administration of amoxicillin/clavulanate potassium and metronidazole, and the use of antiseptic (chlorhexidine 0,2\% mouthwashes) (as previously described ${ }^{[6]}$ ). For all the patients, the surgical protocol included: (1) chlorhexidine $0.2 \%$ mouthwash $30 \mathrm{~mL}$ swished up to $60 \mathrm{~s}$; (2) local anaesthesia, achieved using 3\% mepivacaine hydrochloride without adrenaline; (3) elevation of a full-thickness mucoperiosteal flap; (4) tooth luxation and avulsion; (5) alveolar bone specimen collection (preferably from the interradicular septum); (6) debridement of the post-extraction socket and osteoplasty by means of an ultrasonic surgical device (Mectron Piezosurgery Device®, Mectron Medical Technology, Carasco, Italy); (7) irrigation of the sockets with rifamycin sodium; (8) tension-free soft tissue closure.

\section{Histological assessment}

Specimens were evaluated as previously described by Nicolatou-Galitis et al. ${ }^{[5]}$ The assessment of the glass slides was performed by a general pathologist, who is specialized in oral diseases.

\section{Statistical Analysis}

The statistical units are the patients who satisfy the inclusion criteria of the study. Descriptive statistics of all data were conventionally calculated.

\section{Results:}

Ten cancer patients completed the protocol and were available for the analysis. They were mostly males (60\%), with a mean age $66 \pm 14$ years. Multiple myeloma was the most common diagnosis (30\%), followed by lung (20\%) and breast cancer (20\%).

Overall, the mean duration of ONJ-related medication was $13.3 \pm 5.3$ months. In detail, denosumab was administered to 5 $(50 \%)$, the mean duration of therapy was $13.6 \pm 6.6$ months. Zoledronate was administered to 4 of them (40\%), the mean duration of therapy was $13.3 \pm 4$ months. Bevacizumab was administered to the last patient, the duration of the therapy was 12 months.

Thirteen tooth extractions were made: eight extractions (61.5\%) were from the maxilla and five (38,5\%) from the mandible. Most of the extracted teeth were molars (84,6\%). Dental extraction was indicated for 7 teeth due to Miller degree 3 mobility; five teeth were extracted because of large carious lesions that could not be treated conservatively; two teeth were extracted for pericoronitis.

Histological findings of ONJ were observed in all harvested samples.

\section{Conclusions:}

This outcome may highlight that the proof of non-exposed ONJ might be the histopathologic confirmation of necrotic bone, as stated by European task force on MRONJ[7]. Additionally, alveolar biopsy should possibly be taken in every case of suspected ONJ, which needs a proper and prompt management for successful healing. 
References

\section{References}

1. ^Alberto Bedogni, Giuseppina Campisi, Vittorio Fusco. (2018 Jul). Medication related osteonecrosis of the jaw (MRONJ). Definitions.: London: Qeios 2018. doi:10.32388/594095

2. a, b, c, d, e V. Campisi, G; Bedogni, A; Fusco. (2020). Raccomandazioni clinico-terapeutiche sull'osteonecrosi delle ossa mascellari (ONJ) farmaco-relata e sua prevenzione. New Digital Frontiers Srleditor.: Palermo: Palermo University Press. doi:10.19229/9788855091480/01062020. ISBN 978-88-5509-148-0

3. a, bGiuseppina Campisi, Stefano Fedele, Vittorio Fusco, Giuseppe Pizzo, Olga Di Fede, et al. (2014). Epidemiology, clinical manifestations, risk reduction and treatment strategies of jaw osteonecrosis in cancer patients exposed to antiresorptive agents. Futur Oncol. 10(2):257-275. doi:10.2217/fon.13.211PubMed PMID: 24490612

4. a, bS. L. Ruggiero, T. B. Dodson, J. Fantasia, R. Goodday, T. Aghaloo, et al. (2014). American association of oral and maxillofacial surgeons position paper on medication-related osteonecrosis of the jaw - 2014 update. J Oral Maxillofac Surg. 72(10):1938-1956. doi:10.1016/j.joms.2014.04.031PubMed PMID: 25234529

5. a, bOurania Nicolatou-Galitis, Erofili Papadopoulou, Emmanouil Vardas, Maria Kouri, Dimitra Galiti, et al. (2020). Alveolar bone histological necrosis observed prior to extractions in patients, who received bone-targeting agents. Oral Dis. 26(5):955-966. doi:10.1111/odi.13294

6. ^Rodolfo Mauceri, Vera Panzarella, Giuseppe Pizzo, Giacomo Oteri, Gabriele Cervino, et al. (2020). Platelet-Rich Plasma (PRP) in Dental Extraction of Patients at Risk of Bisphosphonate-Related Osteonecrosis of the Jaws: A TwoYear Longitudinal Study. Appl Sci. 10(13):4487. doi:10.3390/app10134487

7. ^Morten Schiodt, Sven Otto, Stefano Fedele, Alberto Bedogni, Ourania Nicolatou-Galitis, et al. (2019). Workshop of European task force on medication-related osteonecrosis of the jaw-Current challenges. Oral Dis. 25(7):1815-1821. doi:10.1111/odi.13160PubMed PMID: 31325201 